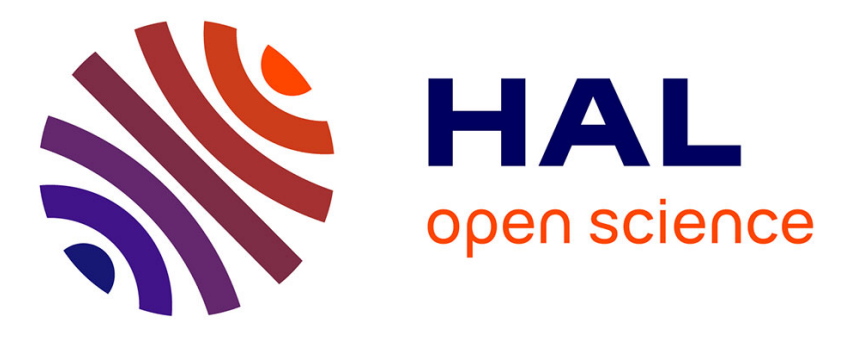

\title{
Implication of nigral dopaminergic lesion and repeated L-dopa exposure in neuropsychiatric symptoms of Parkinson's disease
}

Simon Loiodice, Harry Wing Young, Bertrand Rion, Benoît Méot, Pierre Montagne, Anne-Sophie Denibaud, Roselyne Viel, Christophe Drieu La Rochelle

\section{To cite this version:}

Simon Loiodice, Harry Wing Young, Bertrand Rion, Benoît Méot, Pierre Montagne, et al.. Implication of nigral dopaminergic lesion and repeated L-dopa exposure in neuropsychiatric symptoms of Parkinson's disease. Behavioural Brain Research, 2019, 360, pp.120-127. 10.1016/j.bbr.2018.12.007 . hal-01988160

\section{HAL Id: hal-01988160 \\ https://hal-univ-rennes1.archives-ouvertes.fr/hal-01988160}

Submitted on 31 Jan 2019

HAL is a multi-disciplinary open access archive for the deposit and dissemination of scientific research documents, whether they are published or not. The documents may come from teaching and research institutions in France or abroad, or from public or private research centers.
L'archive ouverte pluridisciplinaire HAL, est destinée au dépôt et à la diffusion de documents scientifiques de niveau recherche, publiés ou non, émanant des établissements d'enseignement et de recherche français ou étrangers, des laboratoires publics ou privés. 
Implication of nigral dopaminergic lesion and repeated L-dopa exposure in neuropsychiatric symptoms of Parkinson's disease

Simon Loiodice*1, Harry Wing Young ${ }^{1}$, Bertrand Rion ${ }^{1}$, Benoît Méot ${ }^{1}$, Pierre Montagne ${ }^{1}$, Anne-Sophie Denibaud $^{1}$, Roselyne Viel ${ }^{2}$, Christophe Drieu La Rochelle ${ }^{1}$

${ }^{1}$ Biotrial Pharmacology, 7-9 rue Jean-Louis Bertrand, 35042 Rennes (France)

${ }^{2}$ Plate-Forme H2P2, Université de Rennes 1, Biosit, 2 Av. du Prof. Léon Bernard, 35043 Rennes (France)

\section{*Corresponding Author:}

\section{Simon Loiodice}

Non-Clinical Department

Biotrial Pharmacology

7-9 rue Jean-Louis Bertrand

35042 Rennes, France

Email: simon.loiodice@biotrial.com

\section{Abstract}

This study aims to investigate the contribution of nigral dopaminergic (DA) cell loss, repeated exposure to DA medication and the combination of both to the development of neuropsychiatric symptoms observed in Parkinson's disease (PD).

A bilateral 6-OHDA lesion of the substantia nigra pars compacta (SNc) was performed in rats. A set of animals was repeatedly administered with L-dopa $(20 \mathrm{mg} / \mathrm{kg} / \mathrm{day})$ and benserazide $(5 \mathrm{mg} / \mathrm{kg} / \mathrm{day})$ over 10 days starting from day 11 post-lesion. Behavioural testing was performed in week 3 postlesion: novel object recognition (NOR), elevated plus maze (EPM) social interaction (SI) tests, and amphetamine-induced hyperlocomotion (AIH).

Immunohistochemical analysis revealed a significant partial lesion (48\%) in 6-OHDA versus sham rats. This lesion was not associated with motor impairment. However, lesioned rats displayed a significant deficit in the NOR, which was reversed by acute treatment with L-dopa/benserazide $(12.5 \mathrm{mg} / \mathrm{kg}$ and 
$15 \mathrm{mg} / \mathrm{kg}$ respectively). Lesioned rats also displayed a deficit in the EPM which was not reversed by acute treatment with L-dopa. No difference was observed in the SI test or in the AlH assay. In all assays, no effect of chronic L-dopa exposure was observed.

This study provides new insights into the neuropathophysiology associated with neuropsychiatric symptoms of PD. Our data strongly emphasises a not previously clearly identified critical role in cognition for the SNc. The results suggest that DA pathways were less directly involved in lesioninduced anxiety-like behaviour. We did not report any effect of chronic L-dopa exposure in the context of partial nigral cell loss.

Keywords: Parkinson's disease; L-dopa; 6-OHDA; neuropsychiatric symptoms

\section{Introduction}

While the discovery of a disease-modifying drug is a major challenge in Parkinson's disease (PD) research, there is still also an urgent unmet need to improve symptom relief in patients (Meissner et al., 2011). In addition to motor impairments, which constitute the cardinal symptoms of PD, various neuropsychiatric manifestations, such as cognitive impairment, psychosis, anxiety, depression, apathy and impulsive/compulsive disorders are observed (Magnard et al., 2016; Meissner et al., 2011). It has been suggested that some of these symptoms may stem from the loss of nigral dopaminergic (DA) neurons (Carnicella et al., 2014; Drui et al., 2013; Magnard et al., 2016; McFarland et al., 2011; Nezhadi et al., 2016). For instance, apathy and affective disorders such as depression and anxiety were investigated in various animal studies, recently reviewed by Magnard and colleagues (Magnard et al., 2016). Despite discrepancies between the studies, it appears that bilateral partial lesion of the substantia nigra pars compacta $(\mathrm{SNC})$ in the rat can result in anxiety-like behavior, motivational deficits, social interaction deficits and depression-like behaviour without any motor impairment (Carnicella et al., 2014; Drui et al., 2013).

The effect of a nigrostriatal lesion on cognitive performance was also investigated in a few studies, reviewed in a report that proposed partial SNc lesioning in the rat as a good model of PD memory disabilities (Da Cunha et al., 2002). More recent studies have generated contradictory data, 
suggesting either a significant impairment in object recognition and/or Morris water-maze assays (Nezhadi et al., 2016; Tadaiesky et al., 2008) or no change (Branchi et al., 2008; Carvalho et al., 2013; Pioli et al., 2008) after a nigrostriatal lesion. However, it is important to note that two of these studies relied on the use of a unilateral lesion (Carvalho et al., 2013; Nezhadi et al., 2016). In one case, lesion-induced impairment of general locomotor activity was observed (Carvalho et al., 2013), which may introduce bias when analysing a behavioural outcome that depends on motor performance, such as object exploration or swimming. In the other aforementioned example (Nezhadi et al., 2016), although no impairment of motor performance was observed, one may argue that a unilateral SNc lesion may not sufficiently reproduce all of the compensatory mechanisms (e.g. post-synaptic plasticity) that potentially underlie the cognitive deficit observed in humans undergoing bilateral DA cell loss.

Hallucination and other psychotic disorders observed in PD have been challenging to investigate in animal models, given the subjective nature of hallucinations and the difficulty of inferring meaning from an animal's behaviour (Goldman et al., 2011). Although animal models were developed to investigate schizophrenic psychosis (Janhunen et al., 2015), there are a very limited number of data regarding animal models of PD-related psychosis (especially in the rat). Nevertheless, a study has reported psychosis-like behaviour in rats after bilateral lesion of the SNc (McFarland et al., 2011). In this study, animals were shown to exhibit an enhanced response to psychostimulants (DOI-induced head twitches and amphetamine-induced hypercolocomotion), as well as a deficit in acoustic prepulse inhibition. Other models, such as MPTP-lesioned primates, have been developed and, quite interestingly, psychotic-like behaviour was observed in this type of model only after dopaminergic drug treatment (Titova et al., 2017; Visanji et al., 2006).

Altogether, the aforementioned studies suggest that a nigral lesion per se may underlie a deficit in affective behaviour, as well as cognition, and may induce psychosis-like behaviour. But, although extremely valuable, these studies do not assess the impact of repeated exposure to DA replacement 
therapy (DRT) on neuropsychiatric manifestations of PD. However, previous data reported that chronic exposure to DRT (especially L-dopa) was responsible for aberrant striatal neuroplasticities such as up-regulation of DA receptors, BDNF and FOSB (Bordet et al., 1997; Brown et al., 2005; Bychkov et al., 2007), which are believed to underlie (at least) motor complications (Bezard et al., 2001), and was also associated with PD-related neuropsychiatric disorders in patients (Beaulieu-Boire and Lang, 2015; Evans and Lees, 2004; Kuzuhara, 2001; Martínez-Fernández et al., 2016; Rascol et al., 2003). In agreement with this idea of a DRT-induced dysregulation of the DA system, animal studies have demonstrated that repeated exposure to DRT was associated with reward bias (Loiodice et al., 2017b, 2017a) and compulsive behaviour after nigrostriatal lesion (Dardou et al., 2017) in a parkinsonian context. In these experiments, the behavioural outcome (pramipexole-induced place preference and compulsive-like behaviour, respectively) was associated with an increase of Fos-like positive cells in the striatum (Dardou et al., 2017; Loiodice et al., 2017a) and the frontal cortex (Dardou et al., 2017), highlighting drug-induced changes in brain regions involved in cognitive/affective information processing. Other studies in 6-hydroxydopamine (6-OHDA)-lesioned rats have also reported that chronic L-dopa may worsen lesion-induced anxiety and depression (Eskow Jaunarajs et al., 2010, 2011, 2012), while it has been recently suggested that chronic L-dopa treatment per se could induce depression-like behaviour, anhedonia and cognitive dysfunction in unlesioned rats, with Fos induction and DA receptor dysregulation in the hippocampus (Hernández et al., 2014). Finally, the fact that MPTP-lesioned primates show psychotic-like behaviour only after dopaminergic drug treatment (Titova et al., 2017) is also in line with this idea of a possible major impact of repeated DRT exposure on the onset of neuropsychiatric complications.

In view of the above findings, we sought to clarify the role of nigral DA cell loss, repeated exposure to L-dopa and the combination of both factors in the development of non-motor behavioural impairments. We have used a lesional model based on nigral 6-OHDA injection in which the motor skills of the animals are preserved (Drui et al., 2013). We hypothesized that repeated exposure to L- 
dopa may promote brain remodelling, in a context of lesion-induced plasticities responsible for cognitive-like and affective-like deficits.

\section{Materials \& Methods}

\section{Animals}

Adult male Sprague-Dawley rats (200-230 g) from Janvier Labs (Saint Berthevin, France) were maintained in a controlled environment (lights on 07:00-19:00, $\pm 22^{\circ} \mathrm{C}$ ) with food and water freely available. They were housed 3-4 per cage. This study was carried out in AAALAC (Association for Assessment and Accreditation of Laboratory Animal Care) facilities in strict accordance with the European Communities Council Directive (2010/63/EU) guidelines for the care of laboratory animals. The protocol was approved by the Biotrial Pharmacology Committee on the Ethics of Animal Experiments "Comité de Réflexion Ethique en Expérimentation Animale" (CR2EA), and in accordance with French Research Ministry regulations. All possible efforts were made to minimise suffering.

\section{Bilateral 6-OHDA lesion}

Anaesthesia was induced and maintained with isoflurane while animals were placed in a stereotaxic frame (David Kopf Instruments, CA, USA). 52 rats received a stereotaxic injection of 6-OHDA (2 $\mu \mathrm{g} / \mu \mathrm{L}$ ) or vehicle (saline containing $0.2 \%$ ascorbic acid) into the SNc. This injection consisted of a $3 \mu \mathrm{L}$ deposit at the following coordinates (from bregma): AP: $-5.4 \mathrm{~mm}$; ML: $\pm 1.8 \mathrm{~mm}$ and DV: $-8.1 \mathrm{~mm}$ (incisor bar at $+3.2 \mathrm{~mm}$ below interaural plane) according to the Paxinos rat brain atlas (Paxinos and Watson, 1998) and previous publications (Carnicella et al., 2014; Drui et al., 2013). 6-OHDA or vehicle was administered at a rate of $0.4 \mu \mathrm{L} /$ minute. To avoid reflux, the needle was maintained at the injection site for 5 minutes after the injection.

To limit damage to noradrenergic neurons, imipramine $(15 \mathrm{mg} / \mathrm{kg}$, ip) was administered $15 \mathrm{~min}$ before 6-OHDA lesioning (Loiodice et al., 2018; Michel et al., 2015). 
Transient starvation states occurred 2-3 days after surgery in a subset of lesioned animals (around 20\%). These animals received supplementation with a high-caloric liquid diet and palatable food for 1-2 weeks. Animals that did not recover $(<5 \%)$ were euthanised for ethical reasons.

\section{Drugs}

All drugs (Sigma, France) were dissolved in saline, except 6-OHDA, which was dissolved in a saline solution containing $0.2 \%$ ascorbic acid.

L-dopa chronic treatment was initiated 10 days after stereotaxic surgery. A subset of SNc-lesioned animals ('Chronic L-dopa: ON' in Fig. 1) received a daily subcutaneous injection of L-dopa methyl ester $20 \mathrm{mg} / \mathrm{kg}$ and benserazide $5 \mathrm{mg} / \mathrm{kg}$ for 12 consecutive days (between 2.00 and $3.00 \mathrm{PM}$ ). This dose was chosen based on previous studies demonstrating L-dopa-induced neuroplasticities in the striatum as well as behavioural changes and sensitization paradigms using a similar regimen (Bordet et al., 1997, 2000; Brown et al., 2005; Bychkov et al., 2007; Loiodice et al., 2017b, 2017a, 2018; Sgambato-Faure et al., 2005; van Kampen and Stoessl, 2003).

In the elevated plus maze (EPM) and novel object recognition (NOR) assays, L-dopa methyl ester and benserazide were administered at 12.5 and $15 \mathrm{mg} / \mathrm{kg}$, ip, respectively. This dose was chosen as it was shown to alleviate 6-OHDA lesion-induced behavioural impairment in a similar context (Drui et al., 2013). The choice of having a group of animals acutely treated with L-dopa/benserazide during the EPM and NOR assays but not in other behavioural assays was made based on a pilot study suggesting a lesion-induced impairment only for in the EPM and NOR tests. Also, a 24h-interval was acknowledged between the EPM and NOR assays to allow elimination of L-dopa/benserazide acutely administered for the EPM (see Fig. 1).

\section{Behavioural procedures}


All of the behavioural procedures were initiated 3 weeks after cerebral injection of 6-OHDA and took place between 9.30 and 12.30 AM. An overview of the study design can be seen in Fig. 1.

\section{General locomotor activity}

Spontaneous locomotor activity was measured using an automated actimetry system. Animals were individually placed into cages $(33 \times 21 \times 18 \mathrm{~cm})$ positioned in an activity meter (Imetronic system, France) allowing automatic recording of horizontal animal displacements through infrared beams for a period of 120 min (number of infrared beams break).

\section{Stepping test}

Forelimb akinesia was assessed as previously described (Paillé et al., 2007). Briefly, the experimenter firmly held the rat's hindquarters while it supported its weight on its forelimb. Then, the experimenter moved the rat forward along the table $(0.9 \mathrm{~m}$ in 5 seconds) three consecutive times per session. All sessions were video-recorded and the number of adjusting steps was counted afterwards by a blinded investigator. For each session, the total score calculated was the sum of the number of adjusting steps observed for the left and right forepaw in the three tests.

\section{Elevated plus maze}

Anxiety-like behaviour was assessed as previously described (File et al., 1993). The apparatus consists of a wooden cross-shaped maze, elevated $66 \mathrm{~cm}$ above the floor. It has two opposite open arms, $45 \mathrm{x}$ $10 \mathrm{~cm}$, and two opposite enclosed arms of the same size, but with $50 \mathrm{~cm}$ high walls. The central square formed by the arms is open. The open arms have $2 \mathrm{~cm}$ high Plexiglas borders in order to avoid any possible falls by the animals. The apparatus is placed in a dark room illuminated only by halogen lamps, oriented towards the ceiling, and giving a uniform, dim light (around 50 lux). 
Each rat was treated with L-dopa methyl ester $(12.5 \mathrm{mg} / \mathrm{kg}$, ip) and benserazide $(15 \mathrm{mg} / \mathrm{kg}$, ip) or vehicle 20 min before being placed in the central square of the plus maze, facing an enclosed arm. Animals were free to explore the EPM for a period of $5 \mathrm{~min}$.

The time spent in the open arms and the time spent in the closed arms was recorded during the 5min test period (animals were considered to be in one arm when all four paws were in the arm). The percentage of time spent in the open arms was calculated as follows: time spent in open arms/(time spent in open arms + time spent in closed arms) $\times 100$.

The EPM was cleaned with water after each animal in order to remove any odour trails left by the previous rats.

\section{Social interaction}

The level of social interaction was assessed according to a previously described protocol (Jenkins et al., 2008; Nakashima et al., 2018). The experimental arena consists of a square wooden box $(80 \times 80 \times 40 \mathrm{~cm})$ painted dark blue. The arena was placed in a dark room illuminated only by halogen lamps, oriented towards the ceiling and giving a uniform, dim light in the box (around 60 lux). The day before the test, the rats were allowed to freely explore the box for $10 \mathrm{~min}$ (habituation). On the day of testing, the animals were placed with a naïve Sprague Dawley rat (Janvier Labs, Saint Berthevin, France) of same body weight in the experimental arena for a 10-min experimental session. For each rat, the total amount of time spent in active non-aggressive social behaviour (i.e. sniffing, following, grooming, kicking, mounting, jumping on, wrestling, boxing, and crawling under and over the partner) was scored by an experimenter unaware of the lesion condition.

The arena was cleaned using water between each trial in order to avoid odour trails left by rats.

\section{Novel object recognition}

Memory performance was assessed using the NOR test. The experimental arena consists of a square wooden box $(60 \times 60 \times 40 \mathrm{~cm})$ painted dark blue with a Plexiglas ${ }^{\circledR}$ floor. The arena was placed in a dark 
room illuminated only by halogen lamps, oriented towards the ceiling and giving a uniform, dim light in the box (around 60 lux).

For each NOR experiment, the rats were subjected to two trials separated by an intertrial interval of 120 min. During the first trial (acquisition trial, T1), the rats were placed in the arena containing 2 identical objects, and the time required by each animal to complete $15 \mathrm{~s}$ of object exploration was determined, with a cut-off time of 5 min. Exploration was considered to be pointing the nose at a distance of less than $2 \mathrm{~cm}$ from the object and/or touching the object and was recorded in real time by a trained experimenter unaware of the lesion/treatment conditions with a timer. For the second trial (testing trial, T2), one of the objects presented in the first trial was replaced with an unknown object (novel object), the rats were placed back in the arena for $3 \mathrm{~min}$, and the time spent in active exploration of the familiar (tF) and novel (tN) objects was recorded.

Animals were treated with L-dopa methyl ester $(12.5 \mathrm{mg} / \mathrm{kg}$, ip) and benserazide $(15 \mathrm{mg} / \mathrm{kg}$, ip) or vehicle 20 min before T1. The difference between the time spent in exploration of the novel object and of the familiar object (Delta=tN-tF) and the recognition index (Delta/(tN+tF) $x$ 100) were calculated.

\section{Amphetamine-induced hyperlocomotion}

Amphetamine-induced hyperlocomotion was measured using an automated actimetry system (as above). Animals were individually placed into cages $(33 \times 21 \times 18 \mathrm{~cm})$ positioned in an activity meter (Imetronic system, France) allowing automatic recording of horizontal animal displacements through infrared beams. After 30 min spent in the actometer, all animals received an intraperitoneal injection of amphetamine $3 \mathrm{mg} / \mathrm{kg}$ (McFarland et al., 2011) and general locomotor activity was recorded over another $90 \mathrm{~min}$.

\section{Histological analysis}


After the last day of behavioural testing, the animals were decapitated under pentobarbital anaesthesia (200 mg/kg, ip) and the brain was quickly removed at $4^{\circ} \mathrm{C}$ on fresh ice. The brains were then post-fixed in ice-cold $4 \%$ paraformaldehyde over $24 \mathrm{~h}$ and processed for paraffin embedding before being sectioned ( $7-\mu \mathrm{m}$ slices) in the coronal plane. The slides were stained using antibodies against tyrosine hydroxylase (TH) (rabbit 1/5000: Millipore AB152) and the DAKO LSAB and HRP system (DAKO Real, DAKO France). Immunostaining was processed on a Discovery XT ${ }^{\circledR}$ Platform (Roche Ventana, Tucson, AZ, USA). All slides were scanned at 20x magnification for whole slide imaging using a NanoZoomer 2.0RS scanner (Hamamatsu, Japan) and analysed using NIS-AR software (Nikon, Japan).

$\mathrm{TH}+$ cells in the SNc were counted at 3 different rostrocaudal levels (AP: $-4.92 \mathrm{~mm},-5.40 \mathrm{~mm}$ and $5.88 \mathrm{~mm}$ ) according to the Paxinos rat brain atlas (Paxinos and Watson, 1998) as previously described (Loiodice et al., 2018; Paillé et al., 2007). At each level, the number of TH+ cells contained in the SNc was counted in three adjacent sections by a blinded experimenter. For each section, the boundaries were chosen by examining the shape of the cells and referring to the Paxinos rat brain atlas (Paxinos and Watson, 1998). At 100x magnification, only the cells with a pyramidal shape were counted. For each animal, the total number of $\mathrm{TH}+$ cells was estimated using the Konigsmark formula: $\mathrm{Nt}=\mathrm{Ns} \mathrm{x}$ (St/Ss) where $\mathrm{Nt=total}$ number of cells; Ns=number of cells counted; St=total number of sections counted; Ss=total number of sections through the SNc (German and Manaye, 1993; Paillé et al., 2007). The percentage of DA cell loss was estimated using the following formula: $100-(\mathrm{TH}+$ cell number in lesioned SNc/TH+cells number in sham SNc $\times 100)$.

\section{Data and statistical analysis}

Data were analysed by Student's t-test, one-way ANOVA, repeated measures ANOVA or two-way repeated measures ANOVA depending on the experimental design. Where appropriate, post-hoc analyses were carried out with Dunnett's test. All reported p-values are two-sided. The normality of the variables was assessed by Shapiro-Wilk test and the homoscedasticity was assessed by Levene's 
test. For each behavioural assay, animals presenting individual values above mean $\pm 2 S D$ were considered outliers and discarded from the analysis.

\section{RESULTS}

Bilateral SNc lesion was associated with a memory deficit and anxiety-like behaviour, which were not enhanced by repeated exposure to L-dopa. Whether they were associated with chronic L-dopa treatment or not, the nigral lesion did not produce a deficit in social interaction or an enhanced response to AlH.

As shown in Fig. $\mathbf{2 a}$ and Fig. 2b, the animals that received a stereotaxic injection of 6-OHDA showed a partial but significant decrease in the number of TH+ cells within the SNc (48.23\% cell loss compared to sham rats, $\left.\mathrm{t}_{28}=6.84 ;{ }^{* *} \mathrm{p}<0.0001\right)$. Fig. $\mathbf{2 c}$ and Fig. $\mathbf{2 d}$ reveal that this partial SNc lesion was not associated with forelimb akinesia or impairment of general locomotor activity.

Fig 3 shows an impairment of memory performance, as well as anxiety-like behaviour after nigral lesion. Memory performance was assessed using the NOR assay. As shown in Fig. 3a, Fig. 3c and Supplementary Data, lesioned rats displayed a significant decrease in delta value $(0.3 \mathrm{~s})$ and recognition index (4.25\%) compared to sham rats (10.3s and $48.6 \%$, respectively, $p<0.0001$ in both cases). Although this deficit was not directly correlated with the percentage of $\mathrm{TH}+$ cell loss (Fig. $\mathbf{3 b}$ and Fig. 3d), it was reversed by a single injection of L-dopa/benserazide $(12.5 \mathrm{mg} / \mathrm{kg}$ and $15 \mathrm{mg} / \mathrm{kg}$ respectively). Repeated exposure to L-dopa did not affect the deficit observed in lesioned rats, with values for delta and recognition index (2.2s and $7.9 \%$, respectively) close to those observed in lesioned rats not exposed to chronic L-dopa ( $0.3 \mathrm{~s}$ and $4.25 \%$, respectively) and significantly different compared to sham ( $p<0.0001$ for both parameters).

A similar pattern was observed for the EPM assay, assessing anxiety-like behaviour. Fig. 3e reveals a decrease (albeit non-significant) in the percentage of time spent in the open arms of the EPM in SNclesioned rats $(5.8 \%)$ compared to sham rats $(14.0 \%, p=0.101)$. No significant correlation with the 
percentage of $\mathrm{TH}+$ cell loss was observed (Fig. $\mathbf{3 f}$ ) and lesioned rats receiving a single injection of Ldopa/benserazide $20 \mathrm{~min}$ before the test did not exhibit any improvement of this slight deficit $(3.15 \%$ of time spent in open arm versus $14.0 \%$ in sham rats, $p=0.0172$ ). As seen for the NOR test, repeated exposure to L-dopa did not affect the behavioural outcome observed in lesioned rats, with the percentage of time spent in the open arms (6.1\%) close to that observed in lesioned rats not exposed to chronic L-dopa (5.8\%), as well as a similar level of difference with sham rats (though nonsignificant, $p=0.106)$.

Unlike the results obtained for the NOR and EPM assays, Fig. 4a shows that the nigral lesion did not induce any change in the level of social interaction regardless of the repeated L-dopa exposure condition. Fig. $\mathbf{4 b}$ reveals that the locomotor response induced by an acute amphetamine challenge was not changed after 6-OHDA lesion either alone or combined with chronic L-dopa treatment. It worth noting that, consistently with results described in Fig. 2d, Fig. $\mathbf{4 b}$ shows no difference in the general locomotor activity of lesioned rats compared to sham rats before amphetamine injection.

\section{DISCUSSION}

In this study, we have evaluated a panel of behaviours and found that bilateral nigral dopaminergic cell loss was associated with a cognitive deficit and anxiety-like behaviour, which were not influenced by repeated exposure to L-dopa. The memory deficit was reversed by an acute challenge with Ldopa, confirming the critical role played by DA. By contrast, anxiety-like behaviour was not alleviated after a single L-dopa administration, suggesting a potential involvement of other pathways in addition to DA. No lesion- or L-dopa-induced changes in social interaction and amphetamine sensitivity were reported.

In the literature, conflicting results can be found regarding the effect of a nigrostriatal lesion on cognitive performance. Some reports have described impairment in memory performance after 6OHDA lesion in the rat (Da Cunha et al., 2002; Nezhadi et al., 2016; Tadaiesky et al., 2008), while 
others reported no change in similar models (Branchi et al., 2008; Carvalho et al., 2013; Pioli et al., 2008). It is important to note that some of these studies relied on the use of a unilateral nigrostriatal lesion (Carvalho et al., 2013; Nezhadi et al., 2016), which may bias data interpretation, as such a lesion is more likely to induce motor impairment (Blandini et al., 2008) and is less clinically relevant (as both hemispheres are affected in PD patients). In our study, we selectively targeted the nigrostriatal pathway using a bilateral partial lesion of the SNc, and highlighted a significant lesioninduced deficit in the NOR test that cannot be attributed to a motor impairment. Consistently with previous studies (Da Cunha et al., 2002; Nezhadi et al., 2016; Tadaiesky et al., 2008), our data provide robust evidences that nigral DA cell loss was associated with a deficit in object memory performance. The reversion of the NOR deficit by a single administration of L-dopa illustrates the critical role of DA in this behavioural outcome. However, other pathways are likely to be involved, as suggested by the lack of correlation between DA cell loss and NOR performance. Indeed, other monoaminergic systems (e.g. noradrenergic and serotoninergic) are known to be impaired in PD (Delaville et al., 2012) and several studies support their potential involvement in non-motor impairments (BonitoOliva et al., 2014; Delaville et al., 2012; Drui et al., 2013; Faggiani et al., 2015; Tadaiesky et al., 2008; Winter et al., 2007). Regarding the discrepancies with other studies reporting a limited impact of nigral 6-OHDA lesion on cognitive performance (Branchi et al., 2008; Pioli et al., 2008), these might be explained by the difference in the type of lesion being used (e.g. injection site, extent of SNc DA cell loss). Furthermore, it worth noting that aforementioned studies and the present work do not rely on the same behavioural assays. Consequently, the 'cognitive subdomains' being assessed may differ.

Numerous studies have investigated anxiety-like behaviour in rat models of PD using 6-OHDA lesions, and contradictory results were recently highlighted by a review covering the topic (Magnard et al., 2016). Our data suggest that bilateral nigral 6-OHDA lesions were associated with anxiety-like behaviour (though this result was less clear than those obtained for the NOR assay). This finding is in line with previous data generated in other 6-OHDA models suggesting the same trend, with a similar amplitude of response and significant (Tadaiesky et al., 2008) or non-significant lesion effects 
(Carvalho et al., 2013). Our data are also in agreement with previous results obtained using the same nigral lesion (Carnicella et al., 2014; Drui et al., 2013), although, surprisingly, we observed a more modest nigral cell loss (48\% versus $70 \%$ ). In these studies, anxiety-like behaviour was reversed by acute treatment with DA agonists (Carnicella et al., 2014) or L-dopa/benserazide (Drui et al., 2013), whereas no alleviation was observed in our study after single administration of L-dopa/benserazide at the same doses. This raises the idea that expression of the behavioural outcome may involve other non-dopaminergic pathways, as further suggested by the lack of correlation with the DA lesion, which was also observed by Drui and colleagues (Drui et al., 2013). Previous studies mentioned above support this assumption (Bonito-Oliva et al., 2014; Delaville et al., 2012; Drui et al., 2013; Faggiani et al., 2015; Tadaiesky et al., 2008; Winter et al., 2007). In fact, it might be proposed that DA pathways were less crucially involved in anxiety-like behaviour than in memory performance, as (i) the deficit was less clear for this behaviour, (ii) it was not reversed by acute L-dopa and (ii) no correlation with the extent of the lesion was identified (this latter point was also true for memory deficit).

Contradictory results have been generated in different 6-OHDA rat models, in which social interaction were shown to be either decreased (Eskow Jaunarajs et al., 2010) or increased (Branchi et al., 2008) after nigrostriatal lesion in the rat. In our experimental conditions, the 6-OHDA nigral lesion was not associated with changes in social interaction. This contrasts with previous data showing a deficit in the same model (Drui et al., 2013). This discrepancy might be explained by the difference in the extent lesion, which was less severe in our study (48\% cell loss versus $~ 70 \%$ ). In our study, the lesion-induced neuroplasticities underlying social interaction impairment may have not been sufficiently pronounced to allow observation of changes in this behaviour. Another point to potentially consider is the strain of rat being used. Indeed, pigmented strains such as Lister-hooded rats were shown to perform better than albino rats in behavioural tasks, including social interaction (Jenkins et al., 2008). It would be of interest to assess whether using pigmented rats may help to clarify the effects of SNc lesioning on social interaction. 
We were not able to find more than one study assessing psychosis-like behaviour in a rat model of PD (McFarland et al., 2011). In this report, the drug pimavenserin (recently approved for treatment of PD-related psychosis) was shown to be effective in behavioural assays typically used to assess the efficacy of antipsychotic medications. Rats with a bilateral 6-OHDA SNc lesion exhibited an augmented amphetamine-induced hyperactivity. In our study, we did not observe this behavioural outcome. This might be (again) possibly explained by the difference in the extent of the lesion, which was stronger in the study from McFarland and colleagues ( 75\%).

In this study, we highlighted a cognitive deficit and, in a lesser extent, an anxiety-like behaviour after SNc lesion with no motor impairment. As the extent of our lesion was only of $\sim 48 \%$, this finding is in line with clinical data suggesting that a panel of non-motor impairment including cognitive deficit and anxiety may be used as prodromal markers of early stages of PD (Postuma and Berg, 2016). Contrary to our initial hypothesis, none of the behaviours investigated in this study was influenced by repeated L-dopa exposure. This finding diverges from previous studies highlighting decreased social interaction and anxiety-like behaviour in 6-OHDA-lesioned rats (Eskow Jaunarajs et al., 2010) or impaired cognitive function in healthy rats (Hernández et al., 2014) following chronic L-dopa. It is also contrasting with published data demonstrating important L-dopa-induced brain remodelling and behavioural changes in a parkinsonian context (Bordet et al., 1997, 2000; Brown et al., 2005; Bychkov et al., 2007; Loiodice et al., 2017b, 2017a, 2018; Sgambato-Faure et al., 2005; van Kampen and Stoessl, 2003). We believe that the key difference between the present study and the aforementioned reports relies on the degree of the nigral lesion (we report a DA cell loss of $48 \%$ in the SNc). It would be of interest to assess the panel of behaviours examined in this report in more severely lesioned animals (without alteration of motor function), and eventually in animals treated for a longer period of time with L-dopa (and/or at a different dose). This regimen may provide further clarifications of the possible role of repeated L-dopa exposure in the impairment of non-motor behaviours. 


\section{CONCLUSION}

Non-motor symptoms of PD have gained increasing interest in the medical and scientific communities over the last decade because of the extent of their negative impact on patients' quality of life. Although recent research has provided useful information, the pathophysiology of neuropsychiatric manifestations remains elusive. This study strongly emphasises a critical role for the SNc in cognition that had not previously been so clearly identified. The results obtained suggest that DA pathways were less directly involved in lesion-induced anxiety-like behaviour. Unlike our initial hypothesis, we did not report any effect of chronic L-dopa exposure examined behaviours. This lack of effect, especially on anxiety-like behaviour, social interaction and psychosis-like behaviour, deserves further investigation.

\section{Conflict of Interest}

The authors declare that they have no conflict of interest.

\section{Acknowledgements}

The authors thank Fiona McAlpine for reviewing the manuscript. 


\section{References}

Beaulieu-Boire, I., Lang, A.E., 2015. Behavioral effects of levodopa. Mov. Disord. Off. J. Mov. Disord. Soc. 30, 90-102. https://doi.org/10.1002/mds.26121

Bezard, E., Brotchie, J.M., Gross, C.E., 2001. Pathophysiology of levodopa-induced dyskinesia: potential for new therapies. Nat. Rev. Neurosci. 2, 577-588. https://doi.org/10.1038/35086062

Blandini, F., Armentero, M.-T., Martignoni, E., 2008. The 6-hydroxydopamine model: News from the past. Parkinsonism Relat. Disord. 14, S124-S129. https://doi.org/10.1016/j.parkreldis.2008.04.015

Bonito-Oliva, A., Masini, D., Fisone, G., 2014. A mouse model of non-motor symptoms in Parkinson's disease: focus on pharmacological interventions targeting affective dysfunctions. Front. Behav. Neurosci. 8, 290. https://doi.org/10.3389/fnbeh.2014.00290

Bordet, R., Ridray, S., Carboni, S., Diaz, J., Sokoloff, P., Schwartz, J.C., 1997. Induction of dopamine D3 receptor expression as a mechanism of behavioral sensitization to levodopa. Proc. Natl. Acad. Sci. U. S. A. 94, 3363-3367.

Bordet, R., Ridray, S., Schwartz, J.C., Sokoloff, P., 2000. Involvement of the direct striatonigral pathway in levodopa-induced sensitization in 6-hydroxydopamine-lesioned rats. Eur. J. Neurosci. 12, 2117-2123.

Branchi, I., D'Andrea, I., Armida, M., Cassano, T., Pèzzola, A., Potenza, R.L., Morgese, M.G., Popoli, P., Alleva, E., 2008. Nonmotor symptoms in Parkinson's disease: investigating early-phase onset of behavioral dysfunction in the 6-hydroxydopamine-lesioned rat model. J. Neurosci. Res. 86, 2050-2061. https://doi.org/10.1002/jnr.21642

Brown, A.M., Deutch, A.Y., Colbran, R.J., 2005. Dopamine depletion alters phosphorylation of striatal proteins in a model of Parkinsonism. Eur. J. Neurosci. 22, 247-256. https://doi.org/10.1111/j.1460-9568.2005.04190.x

Bychkov, E., Ahmed, M.R., Dalby, K.N., Gurevich, E.V., 2007. Dopamine depletion and subsequent treatment with L-DOPA, but not the long-lived dopamine agonist pergolide, enhances activity of the Akt pathway in the rat striatum. J. Neurochem. 102, 699-711. https://doi.org/10.1111/j.1471-4159.2007.04586.x

Carnicella, S., Drui, G., Boulet, S., Carcenac, C., Favier, M., Duran, T., Savasta, M., 2014. Implication of dopamine D3 receptor activation in the reversion of Parkinson's disease-related motivational deficits. Transl. Psychiatry 4, e401-e401. https://doi.org/10.1038/tp.2014.43

Carvalho, M.M., Campos, F.L., Coimbra, B., Pêgo, J.M., Rodrigues, C., Lima, R., Rodrigues, A.J., Sousa, N., Salgado, A.J., 2013. Behavioral characterization of the 6-hydroxidopamine model of Parkinson's disease and pharmacological rescuing of non-motor deficits. Mol. Neurodegener. 8, 14. https://doi.org/10.1186/1750-1326-8-14

Da Cunha, C., Angelucci, M.E.M., Canteras, N.S., Wonnacott, S., Takahashi, R.N., 2002. The lesion of the rat substantia nigra pars compacta dopaminergic neurons as a model for Parkinson's disease memory disabilities. Cell. Mol. Neurobiol. 22, 227-237.

Dardou, D., Reyrolle, L., Chassain, C., Durif, F., 2017. Chronic pramipexole treatment induces compulsive behavior in rats with 6-OHDA lesions of the substantia nigra and ventral tegmental area. Behav. Brain Res. 332, 327-336. https://doi.org/10.1016/j.bbr.2017.06.016

Delaville, C., Chetrit, J., Abdallah, K., Morin, S., Cardoit, L., De Deurwaerdère, P., Benazzouz, A., 2012. Emerging dysfunctions consequent to combined monoaminergic depletions in Parkinsonism. Neurobiol. Dis. 45, 763-773. https://doi.org/10.1016/j.nbd.2011.10.023

Drui, G., Carnicella, S., Carcenac, C., Favier, M., Bertrand, A., Boulet, S., Savasta, M., 2013. Loss of dopaminergic nigrostriatal neurons accounts for the motivational and affective deficits in Parkinson's disease. Mol. Psychiatry. https://doi.org/10.1038/mp.2013.3 
Eskow Jaunarajs, K.L., Angoa-Perez, M., Kuhn, D.M., Bishop, C., 2011. Potential mechanisms underlying anxiety and depression in Parkinson's disease: consequences of I-DOPA treatment. Neurosci. Biobehav. Rev. 35, 556-564. https://doi.org/10.1016/j.neubiorev.2010.06.007

Eskow Jaunarajs, K.L., Dupre, K.B., Ostock, C.Y., Button, T., Deak, T., Bishop, C., 2010. Behavioral and neurochemical effects of chronic L-DOPA treatment on nonmotor sequelae in the hemiparkinsonian rat. Behav. Pharmacol. 21, 627-637. https://doi.org/10.1097/FBP.0b013e32833e7e80

Eskow Jaunarajs, K.L., George, J.A., Bishop, C., 2012. L-DOPA-induced dysregulation of extrastriatal dopamine and serotonin and affective symptoms in a bilateral rat model of Parkinson's disease. Neuroscience 218, 243-256. https://doi.org/10.1016/j.neuroscience.2012.05.052

Evans, A.H., Lees, A.J., 2004. Dopamine dysregulation syndrome in Parkinson's disease. Curr. Opin. Neurol. 17, 393-398.

Faggiani, E., Delaville, C., Benazzouz, A., 2015. The combined depletion of monoamines alters the effectiveness of subthalamic deep brain stimulation. Neurobiol. Dis. 82, 342-348. https://doi.org/10.1016/j.nbd.2015.07.010

File, S.E., Zangrossi, H., Viana, M., Graeff, F.G., 1993. Trial 2 in the elevated plus-maze: a different form of fear? Psychopharmacology (Berl.) 111, 491-494.

German, D.C., Manaye, K.F., 1993. Midbrain dopaminergic neurons (nuclei A8, A9, and A10): threedimensional reconstruction in the rat. J Comp Neurol 331, 297-309. https://doi.org/10.1002/cne.903310302

Goldman, J.G., Vaughan, C.L., Goetz, C.G., 2011. An update expert opinion on management and research strategies in Parkinson's disease psychosis. Expert Opin. Pharmacother. 12, 20092024. https://doi.org/10.1517/14656566.2011.587122

Hernández, V.S., Luquín, S., Jáuregui-Huerta, F., Corona-Morales, A.A., Medina, M.P., Ruíz-Velasco, S., Zhang, L., 2014. Dopamine receptor dysregulation in hippocampus of aged rats underlies chronic pulsatile L-Dopa treatment induced cognitive and emotional alterations. Neuropharmacology 82, 88-100. https://doi.org/10.1016/j.neuropharm.2013.11.013

Janhunen, S.K., Svärd, H., Talpos, J., Kumar, G., Steckler, T., Plath, N., Lerdrup, L., Ruby, T., Haman, M., Wyler, R., Ballard, T.M., 2015. The subchronic phencyclidine rat model: relevance for the assessment of novel therapeutics for cognitive impairment associated with schizophrenia. Psychopharmacology (Berl.) 232, 4059-4083. https://doi.org/10.1007/s00213-015-3954-6

Jenkins, T.A., Harte, M.K., McKibben, C.E., Elliott, J.J., Reynolds, G.P., 2008. Disturbances in social interaction occur along with pathophysiological deficits following sub-chronic phencyclidine administration in the rat. Behav. Brain Res. 194, 230-235. https://doi.org/10.1016/j.bbr.2008.07.020

Kuzuhara, S., 2001. Drug-induced psychotic symptoms in Parkinson's disease. Problems, management and dilemma. J. Neurol. 248 Suppl 3, III28-31.

Loiodice, S., Denibaud, A.-S., Deffains, W., Alix, M., Montagne, P., Seffals, M., Drieu La Rochelle, C., 2018. Validation of a New Scoring Scale for Behavioral Assessment of L-Dopa-Induced Dyskinesia in the Rat: A New Tool for Early Decision-Making in Drug Development. ACS Chem. Neurosci. 9, 762-772. https://doi.org/10.1021/acschemneuro.7b00426

Loiodice, S., McGhan, P., Gryshkova, V., Fleurance, R., Dardou, D., Hafidi, A., Nogueira da Costa, A., Durif, F., 2017a. Striatal changes underlie MPEP-mediated suppression of the acquisition and expression of pramipexole-induced place preference in an alpha-synuclein rat model of Parkinson's disease. J. Psychopharmacol. Oxf. Engl. 31, 1323-1333. https://doi.org/10.1177/0269881117714051

Loiodice, S., Winlow, P., Dremier, S., Hanon, E., Dardou, D., Ouachikh, O., Hafidi, A., Nogueira da Costa, A., Durif, F., 2017b. Pramipexole induced place preference after L-dopa therapy and nigral dopaminergic loss: linking behavior to transcriptional modifications. Psychopharmacology (Berl.) 15-27, 15-27. https://doi.org/10.1007/s00213-016-4430-7 
Magnard, R., Vachez, Y., Carcenac, C., Krack, P., David, O., Savasta, M., Boulet, S., Carnicella, S., 2016. What can rodent models tell us about apathy and associated neuropsychiatric symptoms in Parkinson's disease? Transl. Psychiatry 6, e753. https://doi.org/10.1038/tp.2016.17

Martínez-Fernández, R., Schmitt, E., Martinez-Martin, P., Krack, P., 2016. The hidden sister of motor fluctuations in Parkinson's disease: A review on nonmotor fluctuations. Mov. Disord. Off. J. Mov. Disord. Soc. 31, 1080-1094. https://doi.org/10.1002/mds.26731

McFarland, K., Price, D.L., Bonhaus, D.W., 2011. Pimavanserin, a 5-HT2A inverse agonist, reverses psychosis-like behaviors in a rodent model of Parkinson's disease. Behav. Pharmacol. 22, 681-692. https://doi.org/10.1097/FBP.0b013e32834aff98

Meissner, W.G., Frasier, M., Gasser, T., Goetz, C.G., Lozano, A., Piccini, P., Obeso, J.A., Rascol, O., Schapira, A., Voon, V., Weiner, D.M., Tison, F., Bezard, E., 2011. Priorities in Parkinson's disease research. Nat. Rev. Drug Discov. 10, 377-393. https://doi.org/10.1038/nrd3430

Michel, A., Downey, P., Van Damme, X., De Wolf, C., Schwarting, R., Scheller, D., 2015. Behavioural Assessment of the A2a/NR2B Combination in the Unilateral 6-OHDA-Lesioned Rat Model: A New Method to Examine the Therapeutic Potential of Non-Dopaminergic Drugs. PLOS ONE 10, e0135949. https://doi.org/10.1371/journal.pone.0135949

Nakashima, M., Imada, H., Shiraishi, E., Ito, Y., Suzuki, N., Miyamoto, M., Taniguchi, T., Iwashita, H., 2018. Phosphodiesterase 2A Inhibitor TAK-915 Ameliorates Cognitive Impairments and Social Withdrawal in N-Methyl-d-Aspartate Receptor Antagonist-Induced Rat Models of Schizophrenia. J. Pharmacol. Exp. Ther. 365, 179-188. https://doi.org/10.1124/jpet.117.245506

Nezhadi, A., Sheibani, V., Esmaeilpour, K., Shabani, M., Esmaeili-Mahani, S., 2016. Neurosteroid allopregnanolone attenuates cognitive dysfunctions in 6-OHDA-induced rat model of Parkinson's disease. Behav. Brain Res. 305, 258-264. https://doi.org/10.1016/j.bbr.2016.03.019

Paillé, V., Henry, V., Lescaudron, L., Brachet, P., Damier, P., 2007. Rat model of Parkinson's disease with bilateral motor abnormalities, reversible with levodopa, and dyskinesias. Mov. Disord. 22, 533-539. https://doi.org/10.1002/mds.21308

Paxinos, G., Watson, C., 1998. The Rat Brain in Stereotaxic Coordinates. Academic Press.

Pioli, E.Y., Meissner, W., Sohr, R., Gross, C.E., Bezard, E., Bioulac, B.H., 2008. Differential behavioral effects of partial bilateral lesions of ventral tegmental area or substantia nigra pars compacta in rats. Neuroscience 153, 1213-1224. https://doi.org/10.1016/j.neuroscience.2008.01.084

Postuma, R.B., Berg, D., 2016. Advances in markers of prodromal Parkinson disease. Nat. Rev. Neurol. 12, 622-634. https://doi.org/10.1038/nrneurol.2016.152

Rascol, O., Payoux, P., Ory, F., Ferreira, J.J., Brefel-Courbon, C., Montastruc, J.-L., 2003. Limitations of current Parkinson's disease therapy. Ann. Neurol. 53 Suppl 3, S3-12; discussion S12-15. https://doi.org/10.1002/ana.10513

Sgambato-Faure, V., Buggia, V., Gilbert, F., Lévesque, D., Benabid, A.-L., Berger, F., 2005. Coordinated and spatial upregulation of arc in striatonigral neurons correlates with L-dopa-induced behavioral sensitization in dyskinetic rats. J. Neuropathol. Exp. Neurol. 64, 936-947.

Tadaiesky, M.T., Dombrowski, P.A., Figueiredo, C.P., Cargnin-Ferreira, E., Da Cunha, C., Takahashi, R.N., 2008. Emotional, cognitive and neurochemical alterations in a premotor stage model of Parkinson's disease. Neuroscience 156, 830-840. https://doi.org/10.1016/j.neuroscience.2008.08.035

Titova, N., Schapira, A.H.V., Chaudhuri, K.R., Qamar, M.A., Katunina, E., Jenner, P., 2017. Nonmotor Symptoms in Experimental Models of Parkinson's Disease. Int. Rev. Neurobiol. 133, 63-89. https://doi.org/10.1016/bs.irn.2017.05.018

van Kampen, J.M., Stoessl, A.J., 2003. Effects of oligonucleotide antisense to dopamine D3 receptor mRNA in a rodent model of behavioural sensitization to levodopa. Neuroscience 116, 307314.

Visanji, N.P., Gomez-Ramirez, J., Johnston, T.H., Pires, D., Voon, V., Brotchie, J.M., Fox, S.H., 2006. Pharmacological characterization of psychosis-like behavior in the MPTP-lesioned nonhuman 
primate model of Parkinson's disease. Mov. Disord. Off. J. Mov. Disord. Soc. 21, 1879-1891. https://doi.org/10.1002/mds.21073

Winter, C., von Rumohr, A., Mundt, A., Petrus, D., Klein, J., Lee, T., Morgenstern, R., Kupsch, A., Juckel, G., 2007. Lesions of dopaminergic neurons in the substantia nigra pars compacta and in the ventral tegmental area enhance depressive-like behavior in rats. Behav. Brain Res. 184, 133-141. https://doi.org/10.1016/j.bbr.2007.07.002 


\section{Figure Legends}

Fig. 1. Study design. General experimental procedure and timeline. Blue arrows indicate single acute injection of L-dopa/benserazide (12.5 mg/ $\mathrm{kg}$, ip and $15 \mathrm{mg} / \mathrm{kg}$, ip, respectively) or vehicle. $E P M=e l e v a t e d$ plus maze, NOR=novel object recognition, $\mathrm{SI}=$ social interaction, $\mathrm{AlH}=$ amphetamineinduced hyperlocomotion. A minimum of $24 \mathrm{~h}$-interval was acknowledged between 2 behavioural assays.

Fig. 2. Assessment of nigral loss. (a) Immunolabelling of tyrosine hydroxylase (TH). (b) Number of $\mathrm{TH}+$ cells counted in the SNc in sham and 6-OHDA rats ( $n=9$ and $n=21$, respectively). An unpaired Student's t-test revealed partial DA cell loss in the SNc in 6-OHDA injected rats compared to sham rats $\left(\mathrm{t}_{28}=6.84 ;{ }^{* *} \mathrm{p}<0.0001\right)$. Results are expressed as means $\pm \mathrm{SEM}$. (c) Number of adjusting steps performed with the forepaws $(n=10)$. No significant effect of the lesion was observed for the left $\left(t_{18}=1.17 ; p=0.2574\right)$ or the right paw $\left(t_{18}=0.04997 ; p=0.9601\right)$. Results are expressed as means $\pm S E M$. (d) General locomotor activity ( $n=9-10)$. No significant effect of the lesion was observed $\left(t_{17}=1.181\right.$; $\mathrm{p}=0.254)$. Results are expressed as means \pm SEM.

Fig. 3. Memory performance and anxiety-like behaviour. (a) Delta tN-tF in the NOR assay ( $n=12-13)$. A one-way ANOVA revealed a significant treatment effect, with $F_{3,47}=24.98 ; p<0.0001$. The delta $t N-t F$ was similarly decreased in 6-OHDA-lesioned rats, whether they were chronically treated with Ldopa/benserazide or not, compared to sham rats $(* * * p<0.0001$ with Dunnett's post-hoc). Acute single injection of L-dopa and benserazide (12.5 and $15 \mathrm{mg} / \mathrm{kg}$, ip, respectively) reversed this impairment in 6-OHDA rats not previously exposed to L-dopa (no significant difference compared to sham rats). One (1) 6-OHDA rat untreated (neither chronically nor acutely) with L-dopa exhibited an individual value superior to mean+2SD was considered as outlier and discarded from the analysis. Results are expressed as means \pm SEM. (b) Linear regression revealed no significant correlation between nigral DA cell loss and NOR performance when examining 6-OHDA rats not exposed to Ldopa $(n=10)$. (c) Recognition index in the NOR assay $(n=12-13)$. A one-way ANOVA revealed a significant treatment effect, with $F_{3,47}=26.77 ; p<0.0001$. The recognition index was similarly decreased in 6-OHDA-lesioned rats, whether they were chronically treated with L-dopa/benserazide, or not compared to sham rats $\left({ }^{* *} \mathrm{p}<0.0001\right.$ with Dunnett's post-hoc). Acute single injection of Ldopa and benserazide (12.5 and $15 \mathrm{mg} / \mathrm{kg}$, ip, respectively) reversed this impairment in 6-OHDA rats not previously exposed to L-dopa (no significant difference compared to sham rats). One (1) 6-OHDA rat untreated (neither chronically nor acutely) with L-dopa exhibited an individual value superior to 
mean+2SD was considered as outlier and discarded from the analysis. Results are expressed as means \pm SEM. (d) Linear regression revealed no significant correlation between nigral DA cell loss and NOR performance when examining 6-OHDA rats not exposed to L-dopa $(n=10)$. (e) Ratio of time spent in open arm in the EPM assay ( $n=12-13)$. A one-way ANOVA revealed a significant treatment effect, with $F_{3,47}=3.039 ; p=0.0381$. A decrease was observed in all 6-OHDA-lesioned rats compared to sham. Dunnett's post-hoc analysis revealed that this decrease was significant $(p=0.0172)$ in the acute single injection with L-dopa (12.5 mg/kg, ip) and benserazide (15 mg/kg, ip) condition, but not significant in other lesioned conditions. One (1) 6-OHDA rat untreated (neither chronically nor acutely) with L-dopa exhibited an individual value superior to mean+2SD was considered as outlier and discarded from the analysis. Results are expressed as means \pm SEM. (f) Linear regression revealed no significant correlation between nigral DA cell loss and performance in the EPM assay when examining 6-OHDA rats not exposed to L-dopa $(n=10)$.

Fig. 4. Social interaction and response to amphetamine-induced hyperlocomotion. (a) Level of nonaggressive interaction with a naïve partner $(n=12)$. A one-way ANOVA revealed no significant treatment effect, with $\mathrm{F}_{2,33}=0.506 ; \mathrm{p}=0.607$. One (1) 6-OHDA rat, one (1) 6-OHDA/chronic L-dopa and one (1) sham rat with individual values superior to mean+2SD were considered as outliers and discarded from the analysis. Results are expressed as means \pm SEM. (b) General locomotor activity before and after acute administration of amphetamine ( $3 \mathrm{mg} / \mathrm{kg}$, ip) ( $\mathrm{n}=13)$. A two-way ANOVA revealed a significant time effect $\left(F_{2,276}=19.76 ; p<0.0001\right)$ but no treatment effect $\left(F_{2,24}=0.185\right.$; $p=0.8328)$ and no treatment $x$ time interaction $\left(F_{46,552}=0.9671 ; p=0.5366\right)$. Results are expressed as means \pm SEM. 
FIGURE 1

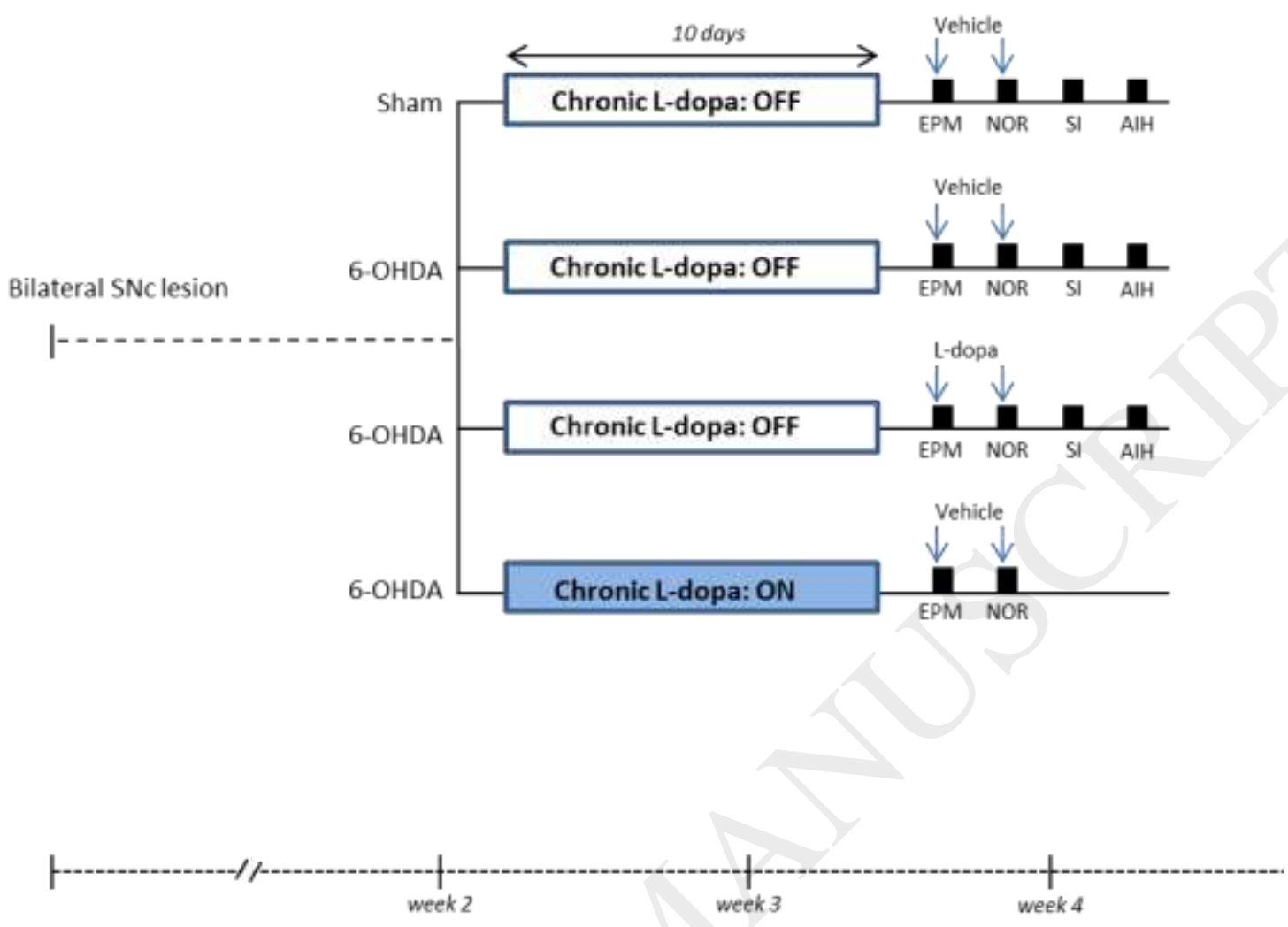


FIGURE 2

a

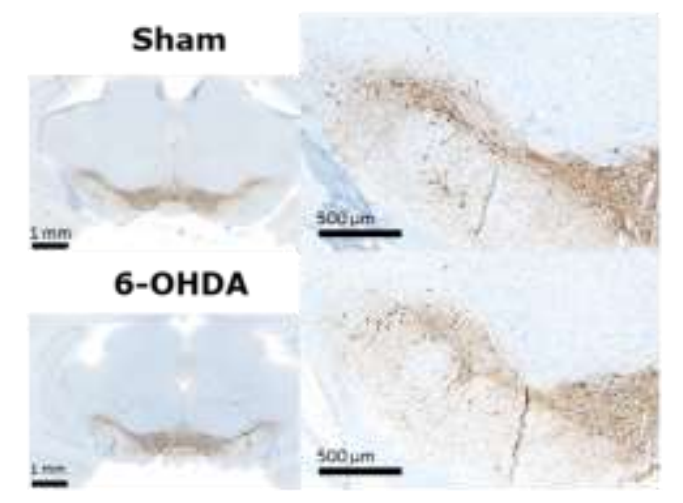

C

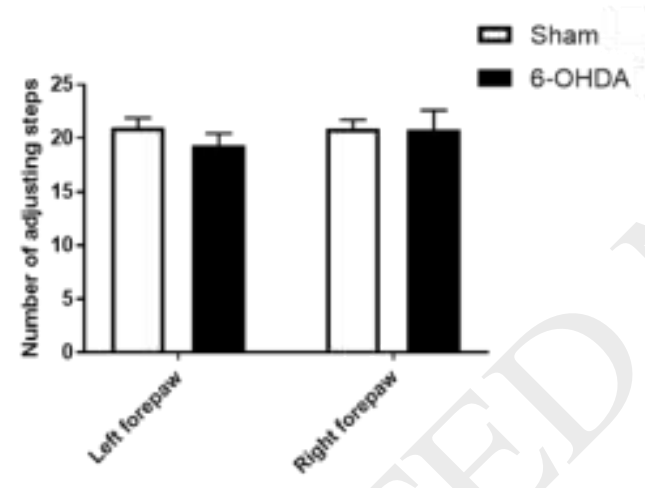

b

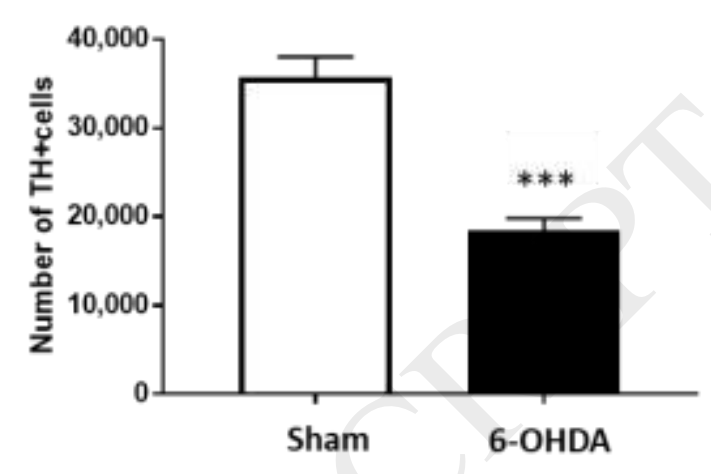

d

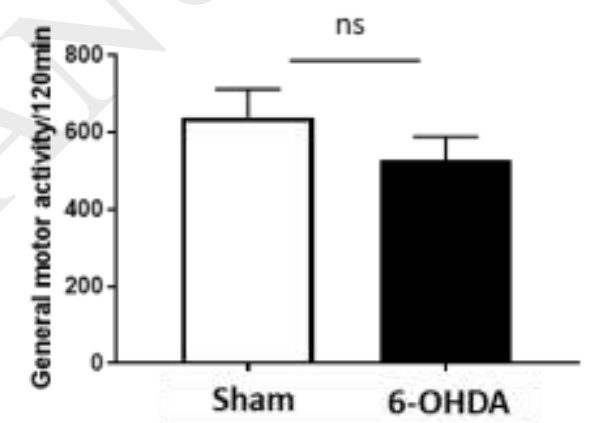


FIGURE 3

a

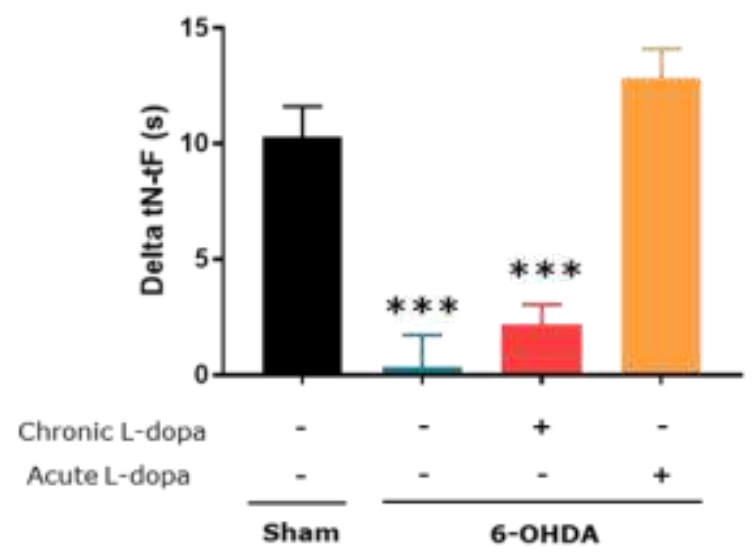

C
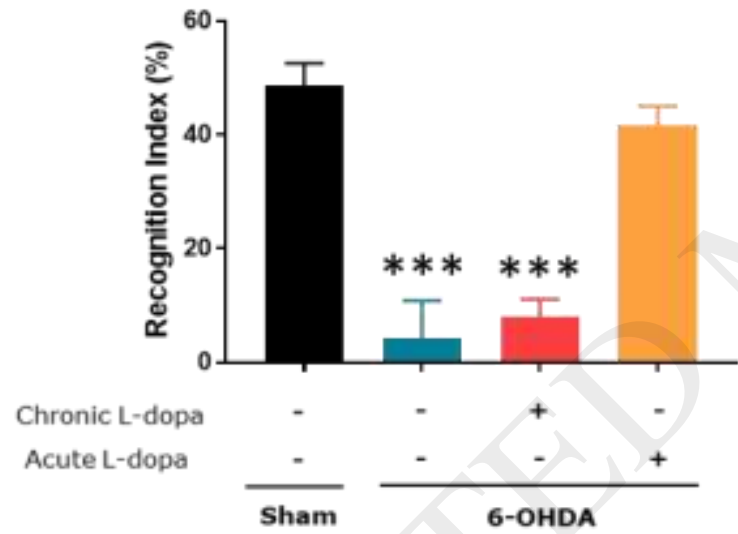

e

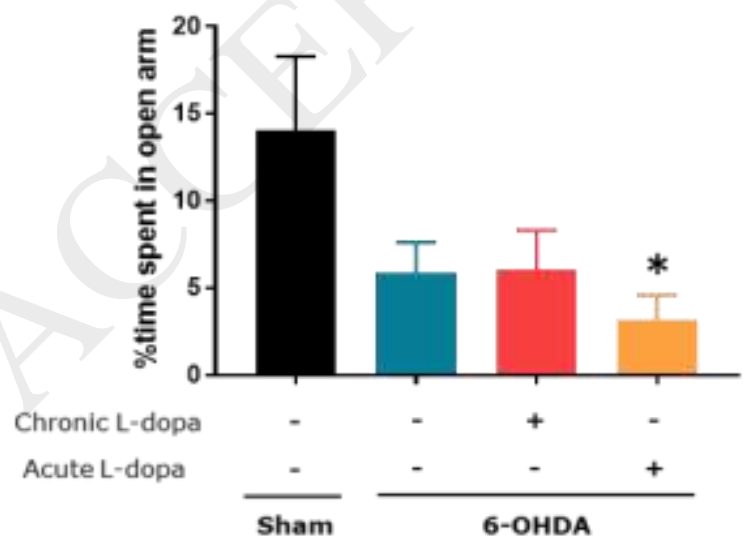

b

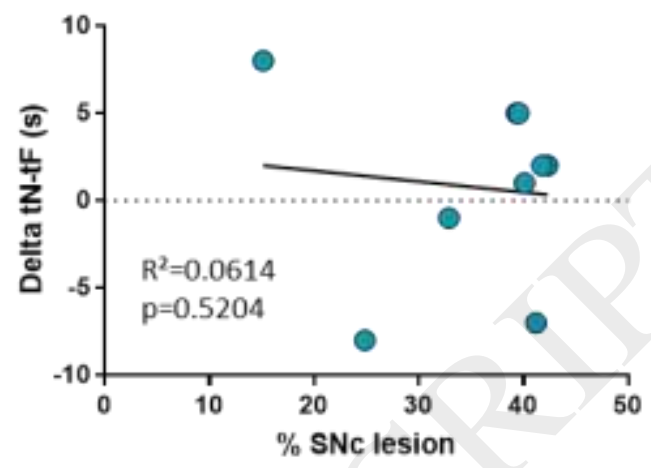

d

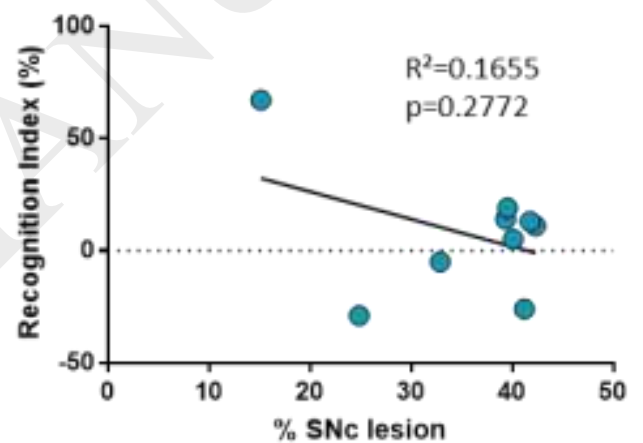

f

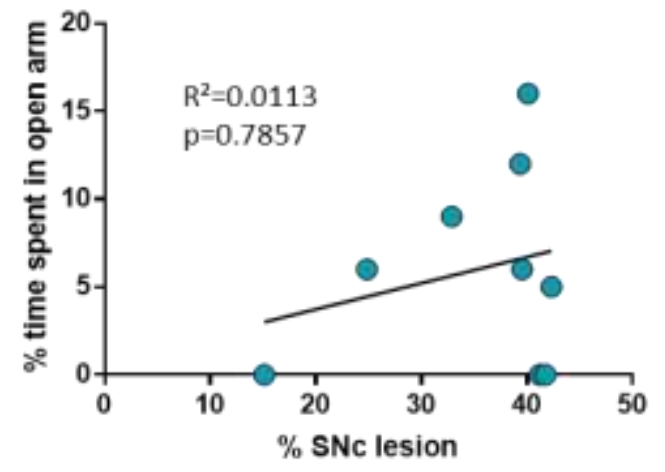


FIGURE 4

a

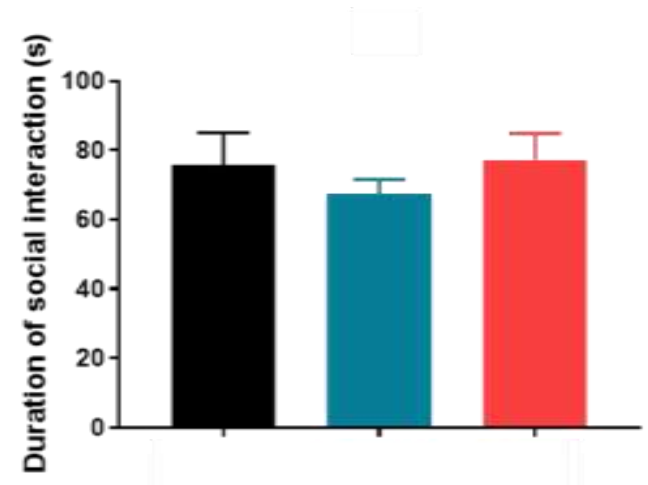

Chronic L-dopa $\frac{-}{\text { Sham }} \stackrel{-}{\text { 6-OHDA }}$

b

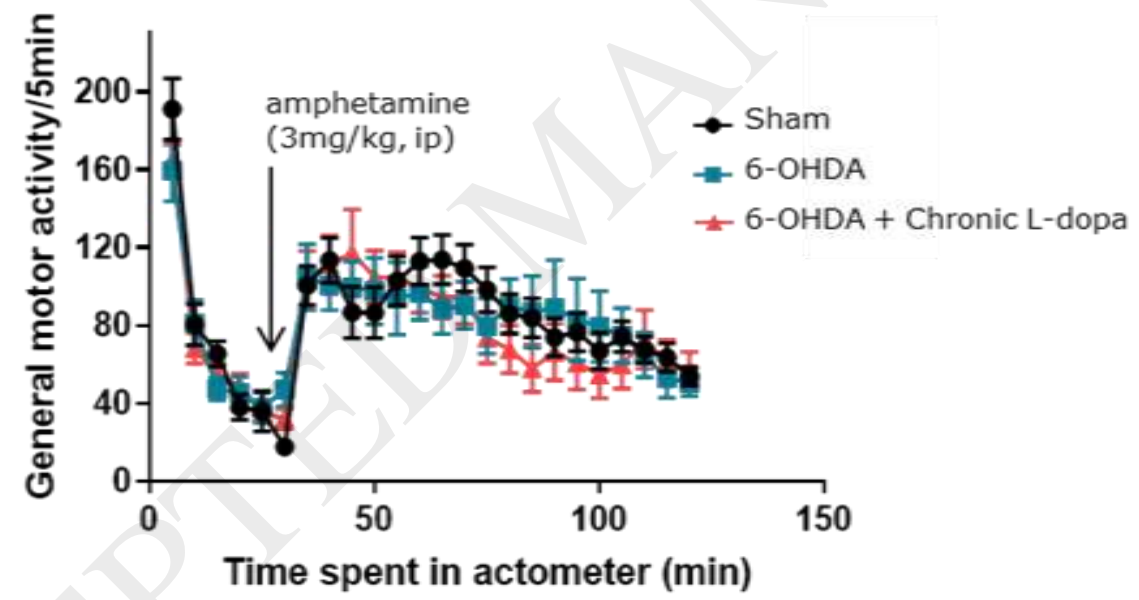

